\title{
Distributions of sparse spanning subgraphs in random graphs
}

\author{
$\mathrm{Pu} \mathrm{Gao}^{*}$ \\ Max-Planck-Institut für Informatik \\ janegao@mpi-inf.mpg.de
}

\begin{abstract}
We describe a general approach of determining the distribution of the number of certain types of spanning subgraphs in the random graph $\mathcal{G}(n, p)$. Using this approach, we reprove the distribution of the number of Hamilton cycles, with a proof that is much shorter than previously known proofs. We also achieve new results on determining the distribution of the number of spanning triangle-free subgraphs and the number of triangle-factors.
\end{abstract}

\section{Introduction}

The distributions of subgraphs with fixed sizes in various random graph models have been investigated by many authors. A general approach by Ruciński [6, 7] showed that the numbers of subgraphs with fixed sizes in the binomial model $\mathcal{G}(n, p)$ are asymptotically normal for a large range of $p$. On the other hand, studies of distributions of subgraphs of sizes growing with $n$, for example, the spanning subgraphs, are much less common. The first breakthrough is perhaps due to Robinson and Wormald [8, 9] on proving that random regular graphs are a.a.s. Hamiltonian. Based on their work, Janson [3] deduced the limiting distribution of the number of Hamilton cycles in random regular graphs. The distributions of some types of spanning subgraphs (perfect matchings, Hamilton cycles, spanning trees) in random graphs $\mathcal{G}(n, p)$ and $\mathcal{G}(n, m)$ were determined by Janson [4]. These distributions behave significantly differently in $\mathcal{G}(n, m)$ and $\mathcal{G}(n, p)$. It was shown that within a big range of $m$, the numbers of these spanning subgraphs are asymptotically normally distributed in $\mathcal{G}(n, m)$, whereas in the corresponding $\mathcal{G}(n, p)$ with $p=m /\left(\begin{array}{l}n \\ 2\end{array}\right)$, these random variables are asymptotically log-normally distributed. This is because the expectations of these variables in $\mathcal{G}(n, m)$ grow very fast as $m$ grows. Therefore, even though the number of edges in $\mathcal{G}(n, p)$ has small deviation, the deviation of these random variables (e.g. the number of perfect matchings) can eventually be very large. This same phenomena was observed by the author [2] while studying the distribution of the number of $d$-factors in $\mathcal{G}(n, p)$.

In this paper, we take the technique that was used in [2] (for the study of $d$-factors) and extend and generalise it into a method for studying a broader class of large (spanning) subgraphs. In Section 2, we describe the general method (Theorems 11 and 3) and give conditions under which the distribution of the random variable under investigation will follow a pattern of concentration

\footnotetext{
*Research supported by the Humboldt Foundation
} 
in $\mathcal{G}(n, m)$ and log-normal distribution in $\mathcal{G}(n, p)$, which we call the log-normal paradigm in this paper. The method is also extended to cope with probability spaces of random directed graphs (See Theorem 5). To show the power of the method, we reprove the distribution of the number of Hamilton cycles. The problem on the number of Hamilton cycles has been studied in the past by a few authors. The first investigation was done by Wright for the directed Hamilton cycles in [11] and then the undirected Hamilton cycles in [10]. Even though both proofs in [11] and [10] are based on a similar counting trick, the analysis for the undirected version is much more complicated. The proof for the directed Hamilton cycles was redone by Frieze and Suen [1], probably unaware of the existing work of Wrignt, using basically the same approach. In [4], Janson reproved the same result for both the undirected and directed versions, using the method of graph decomposition and projection. In this paper, we present a much shorter proof, using our method, for both the directed and undirected versions.

We also present two new results: the distribution of the number of triangle free subgraphs in Section 5, and the distribution of the number of triangle-factors (the spanning subgraphs isomorphic to a collection of vertex disjoint triangles) in Section 6. Their distributions are determined by verifying the conditions given in the theorems in Section 2. In Theorem 1, we state a general approach for proving concentration of any large subgraphs in $\mathcal{G}(n, m)$. The proofs in [2, Theorems 2.3 and 2.4] implicitly follow the approach as described in Theorem 1, though in [2] the setting is only for examining the $d$-factors. The idea of the proof of Theorem 3 is essentially the same as the proof of [4, Theorem 6] (with necessary modifications), except that we relax some constraints in [4, Theorem 6] so that it is applicable to the study of a larger class of subgraphs. The proofs of both Theorems 1 and 3 are presented in Section 7 .

\section{A general approach}

Let $\mathscr{S}$ denote a set of vertex-labelled graphs on a set $S=[n]$ of $n$ vertices. For two graphs $H_{1}$ and $H_{2}$ both on vertex set $S$, let $H_{1} \cap H_{2}\left(H_{1} \cup H_{2}\right)$ denote the set of edges contained in both (either of) $H_{1}$ and $H_{2}$. For any integer $j \geq 0$, let $F_{j}(\mathscr{S})$ denote the set of ordered pairs $\left(H_{1}, H_{2}\right) \in \mathscr{S} \times \mathscr{S}$ such that $\left|H_{1} \cap H_{2}\right|=j$. Let $f_{j}=f_{j}(\mathscr{S})=\left|F_{j}(\mathscr{S})\right|$ and let $r_{j}=f_{j} / f_{j-1}$ for any $j \geq 1$, as long as $f_{j-1} \neq 0$. Let $X_{n}=X_{n}(\mathscr{S})$ denote the number of members of $\mathscr{S}$ that are contained in a random graph $(\mathcal{G}(n, p)$ or $\mathcal{G}(n, m)$, defined on the same vertex set $S$ ) as (spanning) subgraphs. Here $S, p$ and $m$ refer to sequences $(S(n))_{n \geq 1},(p(n))_{n \geq 1}$ and $(m(n))_{n \geq 1}$. Assume every graph in $\mathscr{S}$ has the same number $h=h(n)$ of edges. Let $N(n)=\left(\begin{array}{l}n \\ 2\end{array}\right)$. We drop $n$ from all these notations when there is no confusion. All asymptotics in this paper refer to $n \rightarrow \infty$. For any real $x$ and any integer $\ell \geq 0$, define the $\ell$-th falling factorial $[x]_{\ell}$ to be $\prod_{i=0}^{\ell-1}(x-i)$. Let

$$
\mu_{n}=|\mathscr{S}|\left(\begin{array}{l}
N-h \\
m-h
\end{array}\right) /\left(\begin{array}{l}
N \\
m
\end{array}\right), \quad \lambda_{n}=|\mathscr{S}| p^{h} .
$$

Clearly,

$$
\mathbf{E}_{\mathcal{G}(n, m)} X_{n}=\mu_{n}, \quad \mathbf{E}_{\mathcal{G}(n, p)} X_{n}=\lambda_{n}
$$

A simplification of $\mu_{n}$ (readers can also refer to Lemma 17 by taking $\ell=h$ ) gives

$$
\mathbf{E}_{\mathcal{G}(n, m)} X_{n}=|\mathscr{S}| \cdot \frac{\left(\begin{array}{l}
N-h \\
m-h
\end{array}\right)}{\left(\begin{array}{l}
N \\
m
\end{array}\right)}=|\mathscr{S}| \cdot \frac{[m]_{h}}{[N]_{h}}=|\mathscr{S}|(m / N)^{h} \exp \left(-\frac{N-m}{m N} \frac{h^{2}}{2}+O\left(h^{3} / m^{2}\right)\right) .
$$


Theorem 1 Let $\mu_{n}$ be defined as in (2.1). Assume that $h^{3}=o\left(m^{2}\right), h^{2}=\Omega(m)$ and for $\rho(n)=$ $h^{2} / m$ and some function $\gamma(n)$, the following conditions hold:

(a) for all $K>0$ and for all $1 \leq j \leq K \rho(n)$,

$$
r_{j}=\frac{h^{2}}{N j}\left(1+o\left(m / h^{2}\right)\right) ;
$$

(b) $r_{j} \leq m / 2 N$ for all $4 \rho(n) \leq j \leq \gamma(n)$;

(c) $t(n):=\sum_{j>\gamma(n)} f_{j}=o\left(\mu_{n}|\mathscr{S}|\right)$.

Then in $\mathcal{G}(n, m)$,

$$
X_{n} / \mathbf{E}_{\mathcal{G}(n, m)}\left(X_{n}\right) \stackrel{p}{\rightarrow} 1,
$$

as $n \rightarrow \infty$.

Remark: The ratio $r_{j}$ in condition (a) looks quite restrictive. However, as we will see in the next section, this ratio appears naturally if the edges in $\mathscr{S}$ are distributed randomly (see examples in Sections 3.1 and 3.2). In some cases, for instance, if we take $\mathscr{S}$ to be the set of graphs isomorphic to a given unlabelled graph on $n$ vertices, the edges in $\mathscr{S}$ are likely to still distribute in some kind of "random-like" way and thus having $r_{j}$ as expressed in condition (a) is expected. If we are lucky, we might have condition (b) satisfied for $\gamma(n)=h$. Then $t_{n}=0$ and condition (c) is satisfied trivially. See the example in Section 5. But usually this is not the case, as the sequence $r_{j}$ might decrease first and increase at its tail. Normally, in these cases, condition (c) is not difficult to verify. See examples in Sections 4 and 6 .

Theorem 1 and its proof also gives the following proposition.

Proposition 2 Assume all conditions (a)-(c) of Theorem 1 are satisfied with $m=N$. Then, for all $j=O\left(h^{2} / m\right)$,

$$
f_{j}(n) \sim|\mathscr{S}|^{2} \exp \left(-h^{2} / N\right)\left(h^{2} / N\right)^{j} / j !
$$

The following theorem gives conditions under which $X_{n}$ will be asymptotically log-normally distributed in $\mathcal{G}(n, p)$ if all conditions in Theorem 1 are satisfied by taking $m=p N$.

Theorem 3 Assume $h^{3}=o\left(p^{2} n^{4}\right)$. Let $\beta_{n}=h \sqrt{(1-p) / p N}$ and $\lambda_{n}$ as defined in (2.1). Assume further that $\liminf _{n \rightarrow \infty} \beta_{n}>0$. If for all $m=p N+O(\sqrt{p N}), X_{n} / \mathbf{E}_{\mathcal{G}(n, m)}\left(X_{n}\right) \stackrel{p}{\rightarrow} 1$, then

$$
\frac{\ln \left(e^{\beta_{n}^{2} / 2} X_{n} / \lambda_{n}\right)}{\beta_{n}} \stackrel{d}{\rightarrow} \mathcal{N}(0,1), \quad \text { as } n \rightarrow \infty,
$$

where $\mathcal{N}(0,1)$ is the standard normal distribution.

Combining Theorems 1 and 3 , we immediately have the following corollary. 
Corollary 4 Assume $h^{3}=o\left(p^{2} n^{4}\right)$ and $h^{2}=\Omega\left(p n^{2}\right)$. Let $\beta_{n}=h \sqrt{(1-p) / p N}$ and $\lambda_{n}$ as defined in (2.1). Assume further that $\liminf _{n \rightarrow \infty} \beta_{n}>0$. If for all $m=p N+O(\sqrt{p N})$, conditions (a)-(c) in Theorem 1 are satisfied, then

$$
\frac{\ln \left(e^{\beta_{n}^{2} / 2} X_{n} / \lambda_{n}\right)}{\beta_{n}} \stackrel{d}{\rightarrow} \mathcal{N}(0,1), \quad \text { as } n \rightarrow \infty,
$$

where $\mathcal{N}(0,1)$ is the standard normal distribution.

Hence, in order to prove that a subgraph count has a log-normal distribution in $\mathcal{G}(n, p)$, it is enough to check conditions (a)-(c) in Theorem 1 by taking $m=p N+O(\sqrt{p N})$ if the value of $p$ and the number of edges in the subgraph $h$ satisfy the hypotheses in Corollary 4 . This method is particularly powerful if we can estimate $r_{j}$ without knowing $f_{j}$. This is usually the case when we apply the switching method developed by McKay [5]. As we will see in the later examples, our method is easy to be applied by making extensive use of the switching method.

We can generalise the results to random digraphs. Define $\mathcal{D}(n, m)$ to be the random digraph on $n$ vertices with $m$ directed edges chosen uniformly at random from the $2 N$ ordered pairs of vertices. Define $\mathcal{D}(n, p)$ to be the random digraph on $n$ vertices, which includes every directed edge independently with probability $p$. In this paper, we again define $\mathcal{D}(n, m)$ and $\mathcal{D}(n, p)$ on the vertex set $S$. With almost the same proofs of Theorems 1 and 3 we have the following theorem.

Theorem 5 The same conclusions of Theorems 1 and 3 hold if we replace $\mathcal{G}(n, m), \mathcal{G}(n, p), N$ by $\mathcal{D}(n, m), \mathcal{D}(n, p)$ and $2 N$.

\section{Two trivial examples}

The purpose of this section is to provide simple demonstrations of our method and to convince readers that the behavior of the ratio $r_{j}$ as given in Theorem 1 (a) shall be well expected.

\subsection{The first trivial example}

Take $\mathscr{S}_{1}$ to be the set of all graphs on vertex set $S$ with $h$ edges. Then $\left|\mathscr{S}_{1}\right|=\left(\begin{array}{l}N \\ h\end{array}\right)$. The conclusion of Theorem 1 should hold trivially in this case as $X_{n}\left(\mathscr{S}_{1}\right)$ is constant in $\mathcal{G}(n, m)$ (depending only on $m$ and $h$ ). Nevertheless we verify conditions (a) and (b), also for later use in the next section. For all $0 \leq j \leq h$,

$$
f_{j}=\left(\begin{array}{c}
N \\
j
\end{array}\right)\left(\begin{array}{c}
N-j \\
h-j
\end{array}\right)\left(\begin{array}{c}
N-h \\
h-j
\end{array}\right)
$$

Then for all $1 \leq j \leq h$,

$$
r_{j}=\frac{(h-j+1)^{2}}{j(N-2 h+j)}=\frac{h^{2}}{j N}\left(1+O\left(j / h+h / n^{2}\right)\right) .
$$

This verifies conditions (a) and (b) (for $\gamma(n)=h)$. 


\subsection{Another trivial example}

Let $0<\hat{p}<1$. Consider the set of graphs $\mathscr{S}_{2}$ that is obtained by including each element in $\mathscr{S}_{1}$ independently with probability $\hat{p}$. Note here that $\mathscr{S}_{2}$ itself is a random variable. Then we have the following.

Theorem 6 Assume $0<\hat{p} \leq 1,0<p<1$ are reals and $h$ is an integer that satisfy $m=p\left(\begin{array}{c}n \\ 2\end{array}\right)$, $h^{3}=o\left(m^{2}\right), h^{2}=\Omega(m), m^{2} \hat{p}^{2} N^{h}>>h^{3 h+4} \ln n$. Let $\mu_{n}\left(\mathscr{S}_{2}\right)$ and $\lambda_{n}\left(\mathscr{S}_{2}\right)$ be defined as in (2.1) and let $\beta_{n}=h \sqrt{(1-p) / p N}$. Then $X_{n}\left(\mathscr{S}_{2}\right) / \mu_{n}\left(\mathscr{S}_{2}\right) \stackrel{p}{\rightarrow} 1$ in $G(n, m)$, and

$$
\frac{\ln \left(e^{\beta_{n}^{2} / 2} X_{n}\left(\mathscr{S}_{2}\right) / \lambda_{n}\left(\mathscr{S}_{2}\right)\right)}{\beta_{n}} \stackrel{d}{\rightarrow} \mathcal{N}(0,1), \quad \text { in } \mathcal{G}(n, p),
$$

provided $\liminf _{n \rightarrow \infty} \beta_{n}>0$.

Proof. By the definition of $\mathscr{S}_{2}$, we have $s_{2}=\left|\mathscr{S}_{2}\right| \sim \mathcal{B}\left(\left(\begin{array}{l}N \\ h\end{array}\right), \hat{p}\right)$ and for all $0 \leq j<h, f_{j} \sim$ $\mathcal{B}\left(M_{j}, \hat{p}^{2}\right), f_{h} \sim \mathcal{B}\left(M_{h}, \hat{p}\right)$, where $M_{j}=\left(\begin{array}{c}N \\ j\end{array}\right)\left(\begin{array}{c}N-j \\ h-j\end{array}\right)\left(\begin{array}{c}N-h \\ h-j\end{array}\right)$. Let $A_{n}$ denote the family of $\mathscr{S}_{2}$ which satisfies

$$
\forall 0 \leq j \leq h-1, f_{j}=\left(1+o\left(m / h^{2}\right)\right) \hat{p}^{2} M_{j}, \quad f_{h}<2 M_{h} \hat{p}, \quad s_{2}>\left(\begin{array}{c}
N \\
h
\end{array}\right) \hat{p} / 2 .
$$

The Chernoff bound gives that

$$
\mathbf{P}\left(\left|f_{j}-\hat{p}^{2} M_{j}\right|>2 \sqrt{3(\ln n) \hat{p}^{2} M_{j}}\right)<\exp (-3 \ln n)=n^{-3}, \quad \forall 0 \leq j \leq h-1,
$$

and

$$
\mathbf{P}\left(f_{h}<2 M_{h} \hat{p}\right)=1-o(1), \quad \mathbf{P}\left(s_{2}>\left(\begin{array}{c}
N \\
h
\end{array}\right) \hat{p} / 2\right)=1-o(1) .
$$

Therefore, with probability at least $1-h n^{-3}-o(1)=1-o(1)$, for all $0 \leq j \leq h-1$,

$$
f_{j}=\left(1+O\left(\sqrt{\ln n / \hat{p}^{2} M_{j}}\right)\right) \hat{p}^{2} M_{j} .
$$

Note that for all $j, M_{j}>[N]_{h} /(h !)^{3}>\left(N / h^{3}\right)^{h}$ and $\hat{p}$ satisfies

$$
\hat{p}^{2}>>\frac{h^{4+3 h} \ln n}{m^{2} N^{h}} .
$$

Thus, a.a.s. for all $0 \leq j \leq h-1$,

$$
f_{j}=\left(1+o\left(m / h^{2}\right)\right) \hat{p}^{2} M_{j},
$$

i.e. $\mathbf{P}\left(S_{2} \in A_{n}\right)=1-o(1)$. For every $S_{2} \in A_{n}$, by the calculations in Section 3.1, both conditions (a) and (b) (for $\gamma(n)=h-1$ ) are satisfied whereas condition (c) can be easily verified by noting that $t_{n}=f_{h}<2 M_{h} \hat{p}=o\left(\mu_{n}\left|\mathscr{S}_{2}\right|\right)$. The theorem thereby follows.

The following is a corollary of Theorem [ by letting $\hat{p}=1 / 2$. Here $\mathscr{S}_{2}^{\prime}$ are no longer random variables. We may consider $\mathscr{S}_{2}^{\prime}$ as elements in $A_{n}$ in the proof of Theorem 6 .

Corollary 7 Assume $0<p<1$ is a real and $h$ is an integer that satisfy $m=p\left(\begin{array}{l}n \\ 2\end{array}\right), h^{3}=o\left(m^{2}\right)$, $h^{2}=\Omega(m), m^{2} N^{h}>>h^{3 h+4} \ln n$. Then for almost all subsets $\mathscr{S}_{2}^{\prime}$ of $\mathscr{S}_{1}$, the same conclusions of Theorem [6 hold when $\mathscr{S}_{2}$ is replaced by $\mathscr{S}_{2}^{\prime}$. 


\section{A new approach - Hamilton cycles}

The most interesting examples of $\mathscr{S}$ are perhaps taking $\mathscr{S}$ as the set of graphs that are isomorphic to a given unlabelled graph $H$ on a set of $n$ vertices. In this section, we investigate the number of Hamilton cycles. In literature, computing the second moment of the number of Hamilton cycles involves heavy analysis, as done by Wright [10, 11, using the inclusion and exclusion and some recursive functions, and by Janson [4], using the graph decompostion and projection. Here, we present a new and much shorter proof.

Let $H\left(H^{\prime}\right)$ be a cycle (directed cycle) with length $n$ and $\mathscr{S}_{3}\left(\mathscr{S}_{3}^{\prime}\right)$ to be the set of graphs (directed graphs) on $S$ that are isomorphic to $H\left(H^{\prime}\right)$. Thus, $X_{n}\left(\mathscr{S}_{3}\right)$ and $X_{n}\left(\mathscr{S}_{3}^{\prime}\right)$ count the numbers of undirected and directed Hamilton cycles respectively. It is well known that

$$
\left|\mathscr{S}_{3}\right|=(n-1) ! / 2 \text {, and }\left|\mathscr{S}_{3}^{\prime}\right|=(n-1) !
$$

We have the following theorem for the undirected version.

Theorem 8 Let $0<p<1$ be a real and $0<m<N$ an integer satisfying $m=p N$ and $p>>n^{-1 / 2}$. Let $X_{n}$ denote the number of Hamilton cycles in $\mathcal{G}(n, m)$ (or $\left.\mathcal{G}(n, p)\right)$. Let $\mu_{n}=\mathbf{E}_{\mathcal{G}(n, m)} X_{n}$ and let $\lambda_{n}=\mathbf{E}_{\mathcal{G}(n, p)} X_{n}$. Then $X_{n} / \mu_{n} \stackrel{p}{\rightarrow} 1$ in $G(n, m)$. Assume further that $\lim _{\sup _{n \rightarrow \infty}} p(n)<1$, then

$$
\frac{\ln \left(e^{\beta_{n}^{2} / 2} X_{n} / \lambda_{n}\right)}{\beta_{n}} \stackrel{d}{\rightarrow} \mathcal{N}(0,1), \quad \text { in } \mathcal{G}(n, p),
$$

where $\beta_{n}=\sqrt{2(1-p) / p}$.

Proof. We define two switching operations as follows.

h-switching: Choose an edge $x y \in G_{1} \cap G_{2}$. Then choose edges $x_{1} y_{1} \in G_{1} \backslash G_{2}, x_{2} y_{2} \in G_{2} \backslash G_{1}$ such that $x y x_{1} y_{1}$ and $x y x_{2} y_{2}$ are in a cyclic order in $G_{1}$ and $G_{2}$ respectively. Replace $x y$ and $x_{1} y_{1}$ by $x x_{1}$ and $y y_{1}$ in $G_{1}$, and replace $x y$ and $x_{2} y_{2}$ by $x x_{2}$ and $y y_{2}$ in $G_{2}$. The $h$-switching is applicable if and only if

(a) the six vertices $x, y, x_{i}$ and $y_{i}$ for $i=1,2$ are all distinct;

(b) the edges $x x_{1}$ and $y y_{1}$ are not in $G_{2}$ and the edges $x x_{2}$ and $y y_{2}$ are not in $G_{1}$.

inverse $h$-switching: Choose a pair of vertices $\{x, y\}$ such that $x y \notin G_{1} \cup G_{2}$. For $i=1,2$, choose $x_{i}$ and $y_{i}$ such that $x x_{i} \in G_{i}$ and $y y_{i} \in G_{i}$ and $x x_{i} y y_{i}$ is in a cyclic order in $G_{i}$. The inverse $h$-switching replaces $x x_{i}$ and $y y_{i}$ by $x y$ and $x_{i} y_{i}$ in $G_{i}$ for $i=1,2$. The operation is applicable if and only if

(a') the six vertices $x, y, x_{i}$ and $y_{i}$ for $i=1,2$ are all distinct;

(b') the edges $x x_{i}$ and $y y_{i}$ are not in $G_{1} \cap G_{2}$ for $i=1,2$;

(c') $x_{1} y_{1} \notin G_{2}$ and $x_{2} y_{2} \notin G_{1}$.

For $g \in F_{j}$, let $N(g)$ be the number of $h$-switchings applicable on $g$. There are $2 j$ ways to choose and label the end vertices of the edge $x y \in G_{1} \cap G_{2}$. For any chosen $x y$, there are $n-j+O(1)$ ways to choose and label the end vertices of the edge $x_{i} y_{i} \in G_{i}$, where $j+O(1)$ accounts for the 
case that $x_{i} y_{i} \in G_{1} \cap G_{2}$ and the case that condition (a) is violated. Thus, a rough estimation of $N(g)$ is $2 j(n-j+O(1))^{2}$. The only miscounts are those $x y$ and $x_{i} y_{i}$ such that condition (b) is violated. Clearly, the miscount due to the violation of condition (b) is $O(j n)$ because for any chosen $x y$, there are exactly two choices for $x_{1} y_{1}$ (equivalently $x_{2} y_{2}$ ), such that either $x x_{1}$ or $y y_{1}$ is in $G_{2}$ (equivalently, either $x x_{2}$ or $y y_{2}$ is in $\left.G_{1}\right)$. Thus, $N(g)=2 j n^{2}\left(1-j / n+O\left(n^{-1}\right)\right)^{2}$.

On the other hand, for $g^{\prime} \in F_{j-1}$, let $N^{\prime}\left(g^{\prime}\right)$ denote the number of inverse $h$-switchings applicable on $g^{\prime}$. There are $n^{2}-O(n)$ ways to choose and label vertices $x$ and $y$ such that $x y \notin G_{1} \cup G_{2}$. For any chosen $x y$, there are two ways to choose $x_{i}$ and $y_{i}$ from $G_{i}$ for $i=1,2$ respectively, such that $x x_{i}, y y_{i} \in G_{i}$ and $x x_{i} y y_{i}$ is in a cyclic order in $G_{i}$. Thus, $N^{\prime}\left(g^{\prime}\right)$ is approximately $4\left(n^{2}-O(n)\right)$. The only miscounts are those choices that violate conditions (a') or (b') or (c'). There are only $O(n)$ choices of $x y$ so that (a') or $\left(c^{\prime}\right)$ can possibly be violated, and there are only $O(j n)$ choices of $x y$ so that (b') can possibly be violated. Therefore, $N^{\prime}\left(g^{\prime}\right)=4 n^{2}(1+O(j / n))$.

Hence for all $1 \leq j \leq n / 2$,

$$
r_{j}=\frac{4 n^{2}}{2 j n^{2}}(1+O(j / n))=\frac{2}{j}(1+O(j / n)),
$$

from which we can easily verify Theorem 1 (a), (b) (for $\gamma(n)=n / 2$ ). The proof will be completed by verifying condition (c). Let $G$ be a Hamilton cycle, and let $\kappa_{j}(G)$ denote the number of Hamilton cycles that share at least $j$ edges with $G$. There are $\left(\begin{array}{l}n \\ j\end{array}\right)$ ways to choose $j$ edges from $G$. These chosen edges form $r \leq j$ disjoint paths. Contract each path into a special vertex. The total number of vertices including these special vertices is then $n-j$. There are $(n-j-1) ! / 2$ Hamilton cycles on these vertices. For every such Hamilton cycles, expand each special vertex by its corresponding path (there are two ways to expand each special vertex). Then each expanded Hamilton cycle corresponds to a Hamilton cycle that shares at least $j$ edges with $G$. Thus, for every $G$,

$$
\kappa_{j}(G) \leq\left(\begin{array}{c}
n \\
j
\end{array}\right) \frac{(n-j-1) !}{2} \cdot 2^{j}<n ! 2^{j} / j !
$$

It is then straightforward to verify that

$$
\sum_{j \geq n / 2} f_{j} \leq\left|\mathscr{S}_{3}\right| n ! 2^{n / 2} /(n / 2) !=o\left(\left|\mathscr{S}_{3}\right| \mu_{n}\right) .
$$

The same proof, with only slight modification of the switchings that cope with directed edges, works for the directed version (Theorem 9).

Theorem 9 If all assumptions with $N, \mathcal{G}(n, p)$ and $\mathcal{G}(n, m)$ replaced by $2 N, \mathcal{D}(n, p)$ and $\mathcal{D}(n, m)$ in Theorem 8 hold, then the same conclusion of Theorem 8 holds (for $\beta_{n}=\sqrt{(1-p) / p}$ by the definition of $\beta_{n}$ in Theorem (3).

\section{5 triangle-free subgraphs}

In this section, we consider another example where $\mathscr{S}_{4}$ is the set of all triangle-free graphs on $S$ with $h$ edges and maximum degree at most $\Delta=\Theta\left(h^{1 / 3}\right)$. Then $X_{n}\left(\mathscr{S}_{4}\right)$ counts the number of triangle-free subgraphs with $h$ edges and maximum degree at most $\Delta$. 
Theorem 10 Let $0<p<1$ be a real and $0<m<N$ an integer satisfying $m=p N, h^{2}=\Omega(m)$, $h^{3}=o\left(m^{2}\right)$ (or equivalently $h^{3}=o\left(p^{2} n^{4}\right)$ ) and $h^{8 / 3}=o\left(p n^{3}\right)$. Assume $\Delta=\Theta\left(h^{1 / 3}\right)$ is an integer. Let $X_{n}$ denote the number of triangle-free subgraphs with $h$ edges and maximum degree at most $\Delta$. Let $\mu_{n}$ and $\lambda_{n}$ be defined as in (2.1) and let $\beta_{n}=h \sqrt{(1-p) / p N}$. Then $X_{n} / \mu_{n} \stackrel{p}{\rightarrow} 1$ in $G(n, m)$, and

$$
\frac{\ln \left(e^{\beta_{n}^{2} / 2} X_{n} / \lambda_{n}\right)}{\beta_{n}} \stackrel{d}{\rightarrow} \mathcal{N}(0,1), \quad \text { in } \mathcal{G}(n, p),
$$

provided $\liminf _{n \rightarrow \infty} \beta_{n}>0$.

Proof. Recall that $F_{j}\left(\mathscr{S}_{4}\right)=\left\{\left(G_{1}, G_{2}\right) \in \mathscr{S}_{4} \times \mathscr{S}_{4}:\left|G_{1} \cap G_{2}\right|=j\right\}$. Consider $j \geq 1$ and the classes $F_{j}\left(\mathscr{S}_{4}\right)$ and $F_{j-1}\left(\mathscr{S}_{4}\right)$. Let $K_{n}$ denote the complete graph on $S$. We define two other switchings operating on $\mathscr{S}_{4} \times \mathscr{S}_{4}$ as follows.

$s_{2}$-switching: Let $x$ be an edge in $G_{1} \cap G_{2}$. Choose $y$ and $z$ from $K_{n} \backslash G_{1} \cup G_{2}$, such that

(a) $G_{1} \cup y$ and $G_{2} \cup z$ are triangle-free;

(b) $y(z)$ is not incident with a vertex with degree equal to $\Delta$ in $G_{1}\left(G_{2}\right)$.

Replace $x$ by $y$ in $G_{1}$ and replace $x$ by $z$ in $G_{2}$.

inverse $s_{2}$-switching: Let $x$ be an edge in $K_{n} \backslash G_{1} \cup G_{2}$ such that

(a') $G_{1} \cup x$ and $G_{2} \cup x$ are triangle-free;

(b') In both $G_{1}$ and $G_{2}, x$ is not incident with a vertex with degree equal to $\Delta$.

Let $y \in G_{1} \backslash G_{2}$ and $z \in G_{2} \backslash G_{1}$. Replace $y$ by $x$ in $G_{1}$ and replace $z$ by $x$ in $G_{2}$.

Clearly, an $s_{2}$-switching converts an element $g \in F_{j}\left(\mathscr{S}_{4}\right)$ to an element $g^{\prime} \in F_{j-1}\left(\mathscr{S}_{4}\right)$ and an inverse $s_{2}$-switching converts an element $g^{\prime} \in F_{j-1}\left(\mathscr{S}_{4}\right)$ to an element $g \in F_{j}\left(\mathscr{S}_{4}\right)$ for some $j \geq 1$. For any $g \in F_{j}\left(\mathscr{S}_{4}\right)$, let $N(g)$ denote the number of $s$-switchings that are applicable on $g$. Note that in both $G_{1}$ and $G_{2}$, the number of vertices with degree equal to $\Delta$ is $O\left(h^{2 / 3}\right)$. There are $j$ ways to choose $x$. Given $x$, the number of ways to choose $y$ and $z$ is $N-O\left(h+n h^{2 / 3}+T_{1}(g)\right)$ and $N-O\left(h+n h^{2 / 3}+T_{2}(g)\right)$ respectively, where $T_{i}(g)$ denotes the number of 2-paths in $G_{i}$, and $O\left(n h^{2 / 3}\right)$ bounds the number of forbidden choices such that $y$ (or $z$ ) is incident to a vertex with degree equal to $\Delta$. Let $T(g)=\max \left\{T_{1}(g), T_{2}(g)\right\}$. Clearly $T(g)=O\left(n \Delta^{2}\right)=O\left(n h^{2 / 3}\right)$. So $N(g)=j\left(N-O\left(n h^{2 / 3}\right)\right)^{2}$. Then $N(g)=j N^{2}\left(1+O\left(h^{2 / 3} / n\right)\right)$. For any $g^{\prime} \in F_{j-1}\left(\mathscr{S}_{4}\right)$, let $N^{\prime}\left(g^{\prime}\right)$ denote the number of inverse $s_{2}^{\prime}$-switchings applicable on $g^{\prime}$. Then $N^{\prime}\left(g^{\prime}\right)=(N-O((2 h-j+1)+$ $\left.\left.n h^{2 / 3}+T\left(g^{\prime}\right)\right)\right)(h-j+1)^{2}=N h^{2}\left(1+O\left(h^{2 / 3} / n+j / h\right)\right)$. Since $\sum_{g \in F_{j}\left(\mathscr{S}_{4}\right)} N(g)=\sum_{g^{\prime} \in F_{j-1}\left(\mathscr{S}_{4}\right)} N^{\prime}\left(g^{\prime}\right)$, we have that for all $j \geq 1$,

$$
r_{j}=\frac{N h^{2}}{j N^{2}}\left(1+O\left(h^{2 / 3} / n+j / h\right)\right)=\frac{h^{2}}{j N}\left(1+o\left(m / h^{2}\right)+O(j / h)\right) .
$$

Note that $O\left(h^{2 / 3} / n\right)=o\left(m / h^{2}\right)$ because $h^{8 / 3}=o\left(p n^{3}\right)$. Next we verify conditions (a) and (b) of Theorem 1, For all $j=O\left(h^{2} / m\right), j / h=O(h / m)=o\left(m / h^{2}\right)$ since $h^{3}=o\left(m^{2}\right)$. Thus

$$
r_{j}=\frac{h^{2}}{j N}\left(1+o\left(m / h^{2}\right)\right)
$$


which verifies condition (a). By (5.1), for all $j \geq 3 h^{2} / m$,

$$
r_{j}=\frac{h^{2}}{j N}(1+o(1))+O(h / N) \leq \frac{m}{2 N},
$$

which verifies condition (b) (for $\gamma(n)=h$ ).

\section{Triangle-factors}

Given a graph $G$ on $n$ vertices where $n$ is a multiple of 3, a subgraph of $G$ consisting of $n / 3$ vertex disjoint triangles is called a triangle-factor of $G$. In this section, we assume $n \equiv 0(\bmod 3)$ and consider $H\left(H^{\prime}\right)$ to be the unlabelled graph on $n$ vertices consisting of $n / 3$ vertex disjoint triangles (directed triangles). Let $\mathscr{S}_{5}\left(\mathscr{S}_{5}^{\prime}\right)$ denote the set of graphs on $S$ that are isomorphic to $H\left(H^{\prime}\right)$. Then $X_{n}\left(\mathscr{S}_{5}\right)$ counts the number of triangle-factors and

$$
\left|\mathscr{S}_{5}\right|=\frac{n !}{6^{n / 3}(n / 3) !}, \quad\left|\mathscr{S}_{5}^{\prime}\right|=\frac{n !}{3^{n / 3}(n / 3) !} .
$$

The following theorem determines the limiting distribution of $X_{n}=X_{n}\left(\mathscr{S}_{6}\right)$.

Theorem 11 Let $0<p<1$ be a real and $0<m<N$ an integer satisfying $m=p N$ and $\liminf _{n \rightarrow \infty} p(n)>0$. Let $X_{n}$ denote the number of subgraphs that are isomorphic to a set of $n / 3$ vertex disjoint triangles. Let $\mu_{n}=\mathbf{E}_{\mathcal{G}(n, m)} X_{n}$ and let $\lambda_{n}=\mathbf{E}_{\mathcal{G}(n, p)} X_{n}$. Then $X_{n} / \mu_{n} \stackrel{p}{\rightarrow} 1$ in $\mathcal{G}(n, m)$. Assume further that $\limsup _{n \rightarrow \infty} p(n)<1$, then

$$
\frac{\ln \left(e^{\beta_{n}^{2} / 2} X_{n} / \lambda_{n}\right)}{\beta_{n}} \stackrel{d}{\rightarrow} \mathcal{N}(0,1), \quad \text { in } \mathcal{G}(n, p),
$$

where $\beta_{n}=\sqrt{2(1-p) / p}$.

Remark: Indeed, the condition of $\liminf _{n \rightarrow \infty} p(n)>0$ can be replaced by $p(n) \geq n^{-\delta}$, for some small constant $\delta$. For instance, we checked that $\delta=1 / 16$ works and there is still room for further improvement. However, $p>>n^{-1 / 2}$ does not seem to be sufficient. For the purpose of a cleaner presentation, we only consider $\liminf _{n \rightarrow \infty} p(n)>0$ in the proof. For readers who are interested in improving the condition of $p$, we give quite tight bounds in Lemmas 13 and 14, and we also point out here that there is plenty of room in the proofs of Lemma [16] and Theorem [11 to improve the range of $p$.

Almost the same proof of the previous theorem, with slight modifications of the switchings defined in the proof of Theorem [11, concerning the directions of edges, yields the following corresponding theorem for the number of directed triangle-factors.

Theorem 12 If all assumptions with $N, \mathcal{G}(n, p)$ and $\mathcal{G}(n, m)$ replaced by $2 N, \mathcal{D}(n, p)$ and $\mathcal{D}(n, m)$ in Theorem 11hold, then the same conclusion of Theorem 11 holds (for $\beta_{n}=\sqrt{(1-p) / p}$ by the definition of $\beta_{n}$ in Theorem 3 ). 
For any $\left(G_{1}, G_{2}\right) \in \mathscr{S}_{6} \times \mathscr{S}_{6}$, the edges in $G_{1}$ and $G_{2}$ can intersect in two ways. We say $e \in G_{1} \cap G_{2}$ is of type 1 if the triangles $T_{i} \in G_{i}$ with $e \in T_{i}$ for $i=1,2$ are distinct. We say $e$ is of type 2 if $T_{1}$ and $T_{2}$ are on the same vertex set.

Let $F_{\ell, t}$ denote the set of $\left(G_{1}, G_{2}\right) \in \mathscr{S}_{6} \times \mathscr{S}_{6}$ such that number of edges in $G_{1} \cap G_{2}$ of type 1 and 2 is $\ell$ and $t$ respectively. Clearly $F_{\ell, t}$ is non-empty only if $t$ is a multiple of 3. Clearly $F_{j}\left(\mathscr{S}_{6}\right)=\cup_{k} F_{j-3 k, 3 k}$. Let $f_{\ell, t}=\left|F_{\ell, t}\right|$. Then $f_{j}=\sum_{k=0}^{\lfloor j / 3\rfloor} f_{j-3 k, 3 k}$.

Lemma 13 For any $t \geq 0$ and $\ell \geq 1$ such that $n-4 \ell-3 t-1>0$ and $n-3 \ell-3 t-12>0$,

$$
\frac{2}{\ell} \frac{(n-4 \ell-3 t-1)^{2}}{(n-3 \ell-3 t)^{2}} \leq \frac{f_{\ell, 3 t}}{f_{\ell-1,3 t}} \leq \frac{2}{\ell} \frac{(n-4 \ell-3 t+4)^{2}}{(n-3 \ell-3 t-12)^{2}} .
$$

Proof. We define two switchings operating on $\mathscr{S}_{6} \times \mathscr{S}_{6}$ as shown in Figure 1, $t_{1}$-switching: Take an edge of type 1 in $G_{1} \cap G_{2}$ and label the end vertices $x$ and $y$. Let $u(v)$ be the vertex that is adjacent to both $x$ and $y$ in $G_{1}\left(G_{2}\right)$. Take a triangle $T_{1}\left(T_{2}\right)$ in $G_{1}\left(G_{2}\right)$ that is distinct from $x y u(x y v)$ which does not contain any edge in $G_{1} \cap G_{2}$. Label the vertices of $T_{1}$ $\left(T_{2}\right)$ as $u_{1} u_{2} u_{3}\left(v_{1} v_{2} v_{3}\right)$. Replace these four triangles in $G_{1} \cup G_{2}$ by $x u u_{1}, y u_{2} u_{3} \in G_{1}$ and $x v v_{1}$, $y v_{2} v_{3} \in G_{2}$. The $t_{1}$-switching is applicable only if $v \notin T_{1}, u \notin T_{2}$ and $T_{1} \cap T_{2}=\emptyset$. See Figure 1 . inverse $t_{1}$-switching: A vertex $x$ is pure if both triangles containing $x$ in $G_{1}$ and $G_{2}$ do not contain any edge in $G_{1} \cap G_{2}$. Choose a pure vertex $x$ and label its neighbours in $G_{1}\left(G_{2}\right)$ as $u$ and $u_{1}(v$ and $v_{1}$ ). Then choose another pure vertex $y$ that is distinct from $x, u_{i}$ and $v_{i}$ for $i=1,2$. Label the neighbours of $y$ in $G_{1}\left(G_{2}\right)$ as $u_{2}$ and $u_{3}\left(v_{2}\right.$ and $\left.v_{3}\right)$. Replace these four triangles under consideration by $x y u, u_{1} u_{2} u_{3} \in G_{1}$ and $x y v, v_{1} v_{2} v_{3} \in G_{2}$.
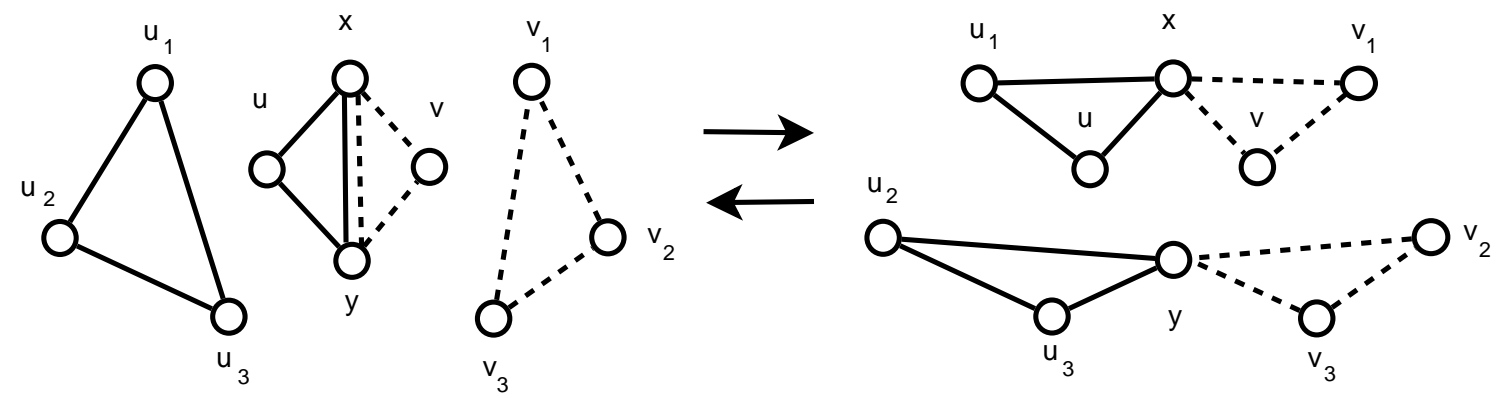

Figure 1: $t_{1}$-switching and its inverse

For any $g=\left(G_{1}, G_{2}\right) \in F_{\ell, 3 t}$, let $N(g)$ be the number of $t_{1}$-switchings that are applicable on $g$. Clearly $N(g) \leq 2 \ell(6(n / 3-(\ell+t)))^{2}$, as there are 2 ways to label $x$ and $y$ for a chosen edge from $G_{1} \cap G_{2}$, and in $G_{1}\left(G_{2}\right)$ there are at most $n / 3-(\ell+t)$ choices for the triangle $u_{1} u_{2} u_{3}\left(v_{1} v_{2} v_{3}\right)$ and for each choice there are 6 ways to label the vertices. We also have

$$
N(g) \geq 2 \ell \cdot 6(n / 3-(\ell+t)-1) \cdot 6(n / 3-(\ell+t)-4),
$$

because for any chosen $x y$, the number of triangles in $G_{1}$ which contain no edges in $G_{2}$ and do not contain $v$ is at least $n / 3-(\ell+t)-1$, whereas given the triangle $u_{1} u_{2} u_{3}$, the number of triangles in $G_{1}$ 
which contain no edges in $G_{1}$ and do not contain any of $u, u_{i}, i=1,2,3$ is at least $n / 3-(\ell+t)-4$. On the other hand, for any $g^{\prime}=\left(G_{1}, G_{2}\right) \in F_{\ell-1,3 t}$, let $N^{\prime}\left(g^{\prime}\right)$ be the number of inverse $t_{1}$-switchings applicable on $g^{\prime}$. The number of pure vertices is exactly $n-4(\ell-1)-3 t$. Hence the number of ways to choose $x$ is $n-4(\ell-1)-3 t$ and for any chosen $x$, the number of ways to label $u, u_{1}, v, v_{1}$ is 4 . The number of ways to choose $y$ is $n-4(\ell-1)-3 t-\delta$, where $\delta$ counts the number of pure vertices among $x, u, u_{1}, v$ and $v_{1}$. Therefore, $1 \leq \delta \leq 5$ always. Hence,

$$
\frac{16(n-4(\ell-1)-3 t-5)^{2}}{2 \ell \cdot(6(n / 3-(\ell+t)))^{2}} \leq \frac{f_{\ell, 3 t}}{f_{\ell-1,3 t}} \leq \frac{16(n-4(\ell-1)-3 t)^{2}}{2 \ell \cdot 36(n / 3-(\ell+t)-4)^{2}} .
$$

Lemma 14 For any $\ell \geq 0$ and $t \geq 1$,

$$
\frac{f_{\ell, 3 t}}{f_{\ell, 3(t-1)}}=\frac{32(n-4 \ell-3 t)^{3}}{3(n-3 \ell-3 t)^{4}}(1+O(1 /(n-4 \ell-3 t))) .
$$

Proof. We define another two switching operations on $\mathscr{S}_{6} \times \mathscr{S}_{6}$ as shown in Figure 2. $t_{2}$-switching: Let $x y z$ be a triangle that is contained in both $G_{1}$ and $G_{2}$. Take two distinct triangles from $G_{1}\left(G_{2}\right)$ which do not contain any edge in $G_{1} \cap G_{2}$ and label the end vertices as $x_{1} y_{1} z_{1}$ and $x_{2} y_{2} z_{2}\left(x_{1}^{\prime} y_{1}^{\prime} z_{1}^{\prime}\right.$ and $\left.x_{2}^{\prime} y_{2}^{\prime} z_{2}^{\prime}\right)$ respectively. Replace the six triangles under consideration by $a a_{1} a_{2} \in G_{1}$ and $a a_{1}^{\prime} a_{2}^{\prime} \in G_{2}$, where $a \in\{x, y, z\}$. This switching is applicable only if all these fifteen vertices $a$, $a_{i}, a_{i}^{\prime}$ for $a \in\{x, y, z\}$ and $i=1,2$ are distinct.

inverse $t_{2}$-switching: Recall from the definition of inverse $t_{1}$-switching that a vertex $x$ is pure if both triangles containing $x$ in $G_{1}$ and $G_{2}$ do not contain any edge in $G_{1} \cap G_{2}$. Choose three pure vertices $a, a \in\{x, y, z\}$ and label the neighbours of $a$ in $G_{1}\left(G_{2}\right)$ by $a_{1}$ and $a_{2}\left(a_{1}^{\prime}\right.$ and $\left.a_{2}^{\prime}\right)$. The inverse $t_{2}$-Switching replaces the six triangles under consideration by $x y z, x_{i} y_{i} z_{i} \in G_{1}$ for $i=1,2$ and $x y z, x_{i}^{\prime} y_{i}^{\prime} z_{i}^{\prime} \in G_{2}$ for $i=1,2$. This switching is applicable only if all these fifteen vertices $a, a_{i}$, $a_{i}^{\prime}$ for $a \in\{x, y, z\}$ and $i=1,2$ are distinct.

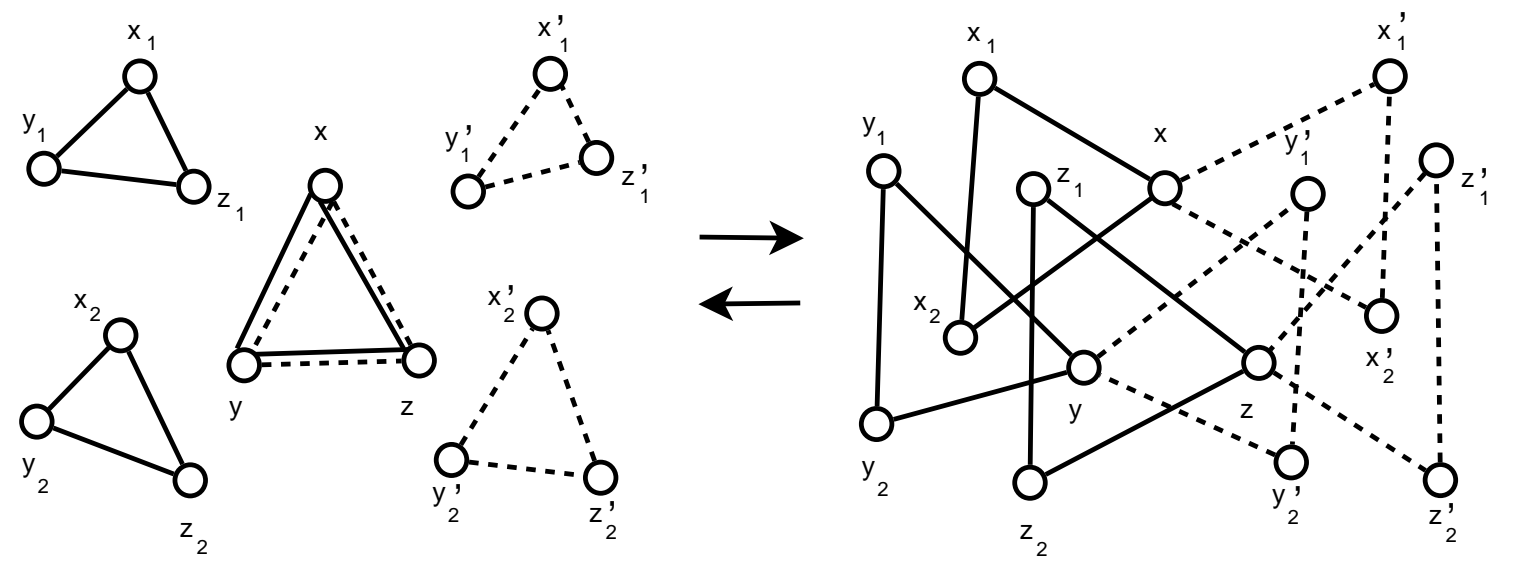

Figure 2: $t_{2}$-switching and its inverse 
For any $g \in F_{\ell, 3 t}$ and $g^{\prime} \in F_{\ell, 3 t-3}$, define $N(g)$ and $N^{\prime}\left(g^{\prime}\right)$ the same way as in the proof of Lemma 13. Following an analogous argument of Lemma 13, it is not hard to show that

$$
\begin{aligned}
& 6 t \cdot 6^{2}\left(\begin{array}{c}
n / 3-(\ell+t)-6 \\
2
\end{array}\right)^{2} \leq N(g) \leq 6 t \cdot 6^{2}\left(\begin{array}{c}
n / 3-(\ell+t) \\
2
\end{array}\right)^{2} \\
& (4(n-4(\ell-1)-3 t-10))^{3} \leq N^{\prime}\left(g^{\prime}\right) \leq(4(n-4(\ell-1)-3 t))^{3} .
\end{aligned}
$$

Thus,

$$
\frac{32(n-4 \ell-3 t-6)^{3}}{3(n-3 \ell-3 t)^{4}} \leq \frac{f_{\ell, 3 t}}{f_{\ell, 3(t-1)}} \leq \frac{32(n-4 \ell-3 t+4)^{3}}{3(n-3 \ell-3 t-21)^{4}}
$$

Corollary 15 For all $j=o(n)$,

$$
\frac{f_{j-3 k-3,3 k+3}}{f_{j-3 k, 3 k}} \sim \frac{4[j-3 k-1]_{3}}{3 n} .
$$

Proof. This follows by Lemmas 13 and 14 and

$$
\frac{f_{j-3 k-3,3 k+3}}{f_{j-3 k, 3 k}}=\frac{f_{j-3 k-3,3 k+3}}{f_{j-3 k-3,3 k}} \prod_{i=0}^{2} \frac{f_{j-3 k-i-1,3 k}}{f_{j-3 k-i, 3 k}} .
$$

Lemma 16 Assume $\liminf _{n \rightarrow \infty} p(n)>0$. Let $\gamma(n)=n / \ln \ln n$. Then

$$
\sum_{j \geq \gamma(n)} f_{j}=o\left(\left|\mathscr{S}_{6}\right| \mu_{n}\right)
$$

Proof. Let $G \in \mathscr{S}_{6}$ and let $\kappa_{j}(G)$ be the number of graphs in $\mathscr{S}_{6}$ which shares at least $j$ edges with $G$. We estimate an upper bound of $\kappa_{j}(G)$. Let $j=\ell+3 t$ and we consider the number of graphs $G^{\prime}$ in $\mathscr{S}_{6}$ that shares at least $\ell$ and $3 t$ edges of type 1 and 2 respectively with $G$. Then there are $\left(\begin{array}{c}n / 3 \\ t\end{array}\right)$ ways to choose the $t$ triangles contained both in $G$ and $G^{\prime}$. Then there are $\left(\begin{array}{c}n / 3-t \\ \ell\end{array}\right) 3^{\ell}$ ways to choose the $\ell$ triangles in $G$ that contain the $\ell$ edges of type 1 and to locate these $\ell$ edges. Given these $\ell$ edges in $G^{\prime}$, there are at most $[n-3 t-2 \ell]_{\ell}$ ways to choose another $\ell$ vertices to form the $\ell$ triangles in $G^{\prime}$. Then there are at most

$$
\frac{(n-3 t-3 \ell) !}{6^{n / 3-t-\ell}(n / 3-t-\ell) !} \leq 9^{n} n^{2(n / 3-t-\ell)}
$$

ways to partition the remaining $n-3 t-3 \ell$ vertices into vertex disjoint triangles in $G^{\prime}$. Hence

$$
\kappa_{j}(G) \leq \sum_{\ell}\left(\begin{array}{c}
n / 3 \\
t
\end{array}\right)\left(\begin{array}{c}
n / 3-t \\
\ell
\end{array}\right) 3^{\ell}[n-3 t-2 \ell]_{\ell} 9^{n} n^{2(n / 3-t-\ell)} \leq n \cdot \max _{\ell}\left\{n^{t} n^{2 \ell} \ell^{-\ell} 9^{n} n^{2(n / 3-t-\ell)}\right\},
$$

where $t=(j-\ell) / 3$. Thus,

$$
\ln \left(\kappa_{j}(G)\right) \leq \max _{\ell}\{(2 n / 3-t) \ln n-\ell \ln (\ell)\}+O(n)
$$


We consider only $j \geq \gamma(n)$. So the maximum is achieved at $\ell=n^{1 / 3}$. Thus

$$
\ln \left(\kappa_{j}(G)\right) \leq \frac{2 n}{3} \ln n-\frac{j}{3} \ln n+O(n),
$$

We also have

$$
\ln \mu_{n}=n \ln p+\frac{2 n}{3} \ln n+O(n) .
$$

So

$$
\ln \left(\kappa_{j}(G)\right)-\ln \mu_{n} \leq-\frac{j}{3} \ln n-n \ln p+O(n) \rightarrow-\infty,
$$

as $n \rightarrow \infty$ since $\liminf _{n \rightarrow \infty} p(n)>0$, which completes the proof of the lemma.

Proof of Theorem 11. For any $j \geq 0$,

$$
r_{j}=\sum_{k=0}^{\lfloor j / 3\rfloor} f_{j-3 k, 3 k} / \sum_{k=0}^{\lfloor(j-1) / 3\rfloor} f_{j-1-3 k, 3 k}
$$

By Corollary 15, for all $j=o\left(n^{1 / 3}\right), r_{j} \sim f_{j, 0} / f_{j-1,0}$. By Lemma 13, this ratio is asymptotic to $2 / j$. This verifies Theorem 1 (a). Let $\gamma(n)=n / \ln \ln n$. Lemma 16 verifies condition (c). The proof is completed by verifying condition (b). Since $r_{j} \sim 2 / j$ for all $j=o\left(n^{1 / 3}\right)$, we only need to show that for all $n^{1 / 3} / \ln n \leq j \leq \gamma(n), r_{j} \leq m / 2 N$. It follows directly from the following two facts.

(a) Let $\widehat{k}=\min \{k: j-3 k \leq \ln n\}$. By Corollary 15.

$$
\sum_{k=0}^{\lfloor j / 3\rfloor} f_{j-3 k, 3 k} \sim \sum_{k=0}^{\widehat{k}} f_{j-3 k, 3 k}, \quad \sum_{k=0}^{\lfloor(j-1) / 3\rfloor} f_{j-1-3 k, 3 k} \sim \sum_{k=0}^{\widehat{k}} f_{j-1-3 k, 3 k} .
$$

(b) By Lemma 13, for all $0 \leq k \leq \widehat{k}, f_{j-3 k, 3 k} / f_{j-1-3 k, 3 k}=o(1)$.

\section{Proofs of Theorems 1 and 3}

Before approaching Theorems 1 and 3, we first prove a technical lemma.

Lemma 17 Let $N=\left(\begin{array}{l}n \\ 2\end{array}\right)$ and let $p=m(n) / N$, where $0<m(n)<N$. Then for any integer $\ell=\ell(n) \geq 0$ such that $\lim _{\sup } \rightarrow \infty(n) / m(n)<1$,

$$
\left(\begin{array}{l}
N-\ell \\
m-\ell
\end{array}\right) /\left(\begin{array}{l}
N \\
m
\end{array}\right)=p^{\ell} \exp \left(-\frac{1-p}{p N} \frac{\ell^{2}-\ell}{2}+O\left(\ell^{3} / m^{2}\right)\right) .
$$

Moreover, if $\ell=\Omega(\sqrt{m})$, then

$$
\left(\begin{array}{l}
N-\ell \\
m-\ell
\end{array}\right) /\left(\begin{array}{l}
N \\
m
\end{array}\right)=p^{\ell} \exp \left(-\frac{1-p}{p N} \frac{\ell^{2}}{2}+O\left(\ell^{3} / m^{2}\right)\right) .
$$




\section{Proof.}

$$
\begin{aligned}
\left(\begin{array}{c}
N-\ell \\
m-\ell
\end{array}\right) /\left(\begin{array}{l}
N \\
m
\end{array}\right) & =\frac{[m]_{\ell}}{[N]_{\ell}}=\prod_{i=0}^{\ell-1} \frac{m-i}{N-i} \\
& =\prod_{i=0}^{\ell-1} \frac{m}{N} \exp \left(-\frac{i}{m}+\frac{i}{N}+O\left(i^{2} / m^{2}\right)\right) \quad\left(\text { since } \limsup _{n \rightarrow \infty} \ell(n) / m(n)<1\right) \\
& =p^{\ell} \exp \left(-\frac{1-p}{p N} \frac{\ell^{2}-\ell}{2}+O\left(\ell^{3} / m^{2}\right)\right) .
\end{aligned}
$$

If we have further that $\ell=\Omega(\sqrt{m})$, then $\ell / p N=O\left(\ell^{3} / m^{2}\right)$.

Proof of Theorem 1. In this proof, the probability space refers to the random graph $\mathcal{G}(n, m)$ only. Let $s=|\mathscr{S}|$. By (2.1) and (2.2),

$$
\mathbf{E} X_{n}=s(m / N)^{h} \exp \left(-\frac{N-m}{m N} \frac{h^{2}}{2}+O\left(h^{3} / m^{2}\right)\right)
$$

We also have

$$
\mathbf{E} X_{n}^{2}=\sum_{j=0}^{h} f_{j}\left(\begin{array}{l}
N-(2 h-j) \\
m-(2 h-j)
\end{array}\right) /\left(\begin{array}{l}
N \\
m
\end{array}\right)
$$

Let $g(j)=f_{j}\left(\begin{array}{l}N-(2 h-j) \\ m-(2 h-j)\end{array}\right) /\left(\begin{array}{l}N \\ m\end{array}\right)$. By condition (a), for every $K>0$ and any $1 \leq j \leq K h^{2} / m$,

$$
\frac{g(j)}{g(j-1)}=r_{j} \cdot \frac{N}{m}(1+O(h / m))=\frac{h^{2}}{m j}\left(1+O(h / m)+o\left(m / h^{2}\right)\right)=\frac{h^{2}}{m j}\left(1+o\left(m / h^{2}\right)\right),
$$

where the last equality holds because $h^{3}=o\left(m^{2}\right)$. By condition (c) and the fact that for any integer $0 \leq j \leq h,\left(\begin{array}{l}N-(2 h-j) \\ m-(2 h-j)\end{array}\right) \leq\left(\begin{array}{l}N-h \\ m-h\end{array}\right)$, we also have that

$$
\sum_{j>\gamma(n)} g(j) \leq t(n)\left(\begin{array}{l}
N-h \\
m-h
\end{array}\right) /\left(\begin{array}{l}
N \\
m
\end{array}\right)=t(n) \mu_{n} / s .
$$

Then for all sufficiently large $K>0$,

$$
\begin{aligned}
\mathbf{E} X_{n}^{2} & =\sum_{j=0}^{h} g(j)=\sum_{j=0}^{K h^{2} / m} g(j)+O\left(g\left(K h^{2} / m\right)\right)+O\left(t(n) \mu_{n} / s\right) \\
& =\left(1+O\left(K^{-1}\right)\right) \sum_{j=0}^{K h^{2} / m} g(j)+O\left(t(n) \mu_{n} / s\right),
\end{aligned}
$$


where the second equality holds because of condition (b) and the last equality holds by (7.1). Next, we estimate $\sum_{j=0}^{K h^{2} / m} g(j)$. By (7.1) and Lemma 17.

$$
\begin{aligned}
& \sum_{j=0}^{K h^{2} / m} g(j)=f_{0} \frac{\left(\begin{array}{c}
N-2 h \\
m-2 h
\end{array}\right)}{\left(\begin{array}{l}
N \\
m
\end{array}\right)} \sum_{j=0}^{K h^{2} / m} \frac{\left(h^{2} / m\right)^{j}}{j !}\left(1+o\left(j m / h^{2}\right)\right) \\
& =f_{0} \cdot(m / N)^{2 h} \exp \left(-\frac{N-m}{m N} \frac{(2 h)^{2}}{2}+O\left(h^{3} / m^{2}\right)\right)\left(\exp \left(h^{2} / m+o(1)\right)+\Gamma(K)\right) \\
& =f_{0} \cdot(m / N)^{2 h} \exp \left(-\frac{N-m}{m N} 2 h^{2}\right) \exp \left(h^{2} / m\right)\left(1+o(1)+O\left(\Gamma(K) \exp \left(-h^{2} / m\right)\right)\right)
\end{aligned}
$$

where

$$
\Gamma(K)=O\left(\frac{\left(h^{2} / m\right)^{K h^{2} / m}}{\left(K h^{2} / m\right) !}\right)=O\left(\left(\frac{\left(e h^{2} / m\right)}{\left(K h^{2} / m\right)}\right)^{K h^{2} / m}\right),
$$

which goes to 0 as $K \rightarrow \infty$, since $h^{2} / m=\Omega(1)$. By (7.2) and (7.3), for every $\epsilon>0$, there is a sufficiently large $K$, such that

$$
\mathbf{E} X_{n}^{2}=(1+O(\epsilon)) f_{0} \cdot(m / N)^{2 h} \exp \left(-\frac{N-m}{m N} 2 h^{2}\right) \exp \left(h^{2} / m\right)+O\left(t(n) \mu_{n} / s\right) .
$$

We also have

$$
s^{2}=\sum_{j=0}^{h} f_{j}=f_{0} \sum_{j=0}^{h} \prod_{i=1}^{j} r_{i}
$$

With the same reasoning as before, it is enough to sum over the first $K h^{2} / N$ terms, leaving an arbitrarily small tail plus an error term $O(t(n))$. This yields

$$
s^{2}=(1+O(\epsilon)) f_{0} \exp \left(h^{2} / N\right)+O(t(n)) .
$$

Since $t(n)=o\left(\mu_{n} s\right)=o\left(s^{2}\right)$ by condition (c), we obtain

$$
f_{0}=(1+O(\epsilon)) s^{2} \exp \left(-h^{2} / N\right)
$$

Combining with (17.4) and again by condition (c), we obtain

$$
\begin{aligned}
\mathbf{E} X_{n}^{2} & =(1+O(\epsilon)) s^{2}(m / N)^{2 h} \exp \left(-\frac{N-m}{m N} 2 h^{2}\right) \exp \left(h^{2} / m-h^{2} / N\right)+O\left(t(n) \mu_{n} / s\right) \\
& =(1+O(\epsilon)) s^{2}(m / N)^{2 h} \exp \left(-\frac{N-m}{m N} h^{2}\right)+o\left(\mu_{n}^{2}\right)=(1+O(\epsilon))\left(\mathbf{E} X_{n}\right)^{2} .
\end{aligned}
$$

As this holds for every $\epsilon>0$, we have $\mathbf{E} X_{n}^{2}=(1+o(1))\left(\mathbf{E} X_{n}\right)^{2}$. Then for every $\epsilon>0$,

$$
\mathbf{P}\left(\left|X_{n} / \mathbf{E} X_{n}-1\right|>\epsilon\right) \rightarrow 0, \text { as } n \rightarrow \infty
$$

by Chebyshev's inequality. 
Proof of Theorem 3. Let $Y_{n}$ denote the number of edges in $\mathcal{G}(n, p)$, then $Y_{n} \sim \operatorname{Bin}(N, p)$. Hence we have

$$
Y_{n}-p N=O_{p}(\sqrt{p(1-p) N}),
$$

where $f(n)=O_{p}(g(n))$ for some $g(n) \geq 0$ means $\mathbf{P}(|f(n)|>K g(n)) \rightarrow 0$ as $K \rightarrow \infty$ and $n \rightarrow \infty$. Similarly we use the notation $f(n)=o_{p}(g(n))$ meaning that for every $\epsilon>0, \mathbf{P}(|f(n)|>\epsilon g(n)) \rightarrow 0$ as $n \rightarrow \infty$. Since $X_{n} / \mathbf{E}_{\mathcal{G}(n, m) X_{n}} \stackrel{p}{\rightarrow} 1$ in $\mathcal{G}(n, m)$ for all $m=p N+O(\sqrt{p(1-p) N})$ by assumption and $\ln \left(\mathbf{E}_{\mathcal{G}(n, m)} X_{n}\right)=\ln |\mathscr{S}|+h \ln (m / N)+(N-m) h^{2} / 2 m N+o(1)$ by (2.2), by conditioning on $Y_{n}$, we have

$$
\ln X_{n}-\ln |\mathscr{S}|-h \ln \left(Y_{n} / N\right)+\frac{1-Y_{n} / N}{Y_{n}} \frac{h^{2}}{2} \stackrel{p}{\rightarrow} 0 .
$$

By (7.5),

$$
\frac{1-Y_{n} / N}{Y_{n}} \frac{h^{2}}{2}=\frac{h^{2}(1-p)}{2 N p}\left(1+O_{p}\left(\sqrt{\frac{p}{(1-p) N}}+\sqrt{\frac{1-p}{p N}}\right)\right)=\frac{h^{2}(1-p)}{2 N p}+o_{p}(1),
$$

where the equality above holds because $h^{3}=o\left(p^{2} n^{4}\right)$. We also have

$$
\ln \left(Y_{n} / N\right)=\ln p\left(1+Y_{n}^{*} \sqrt{(1-p) / p N}\right)=\ln p+\sqrt{(1-p) / p N} Y_{n}^{*}+O_{p}((1-p) / p N),
$$

where

$$
Y_{n}^{*}=\frac{Y_{n}-p N}{\sqrt{p(1-p) N}}
$$

is the normalised variable of $Y_{n}$. Recall that $\lambda_{n}=|\mathscr{S}| p^{h}$ from (2.1) and $\mathbf{E} X_{n}=\lambda_{n}$. Combining with (7.6)-(7.8), we have

$$
\ln \left(X_{n} / \lambda_{n}\right)+\frac{\beta_{n}^{2}}{2}=\beta_{n} Y_{n}^{*}+o_{p}(1)
$$

Since $\beta_{n}=\Omega(1)$, (7.9) immediately yields

$$
\frac{\ln \left(e^{\beta_{n}^{2} / 2} X_{n} / \lambda_{n}\right)}{\beta_{n}}=Y_{n}^{*}+o_{p}(1) .
$$

Since $Y_{n}^{*} \stackrel{d}{\rightarrow} \mathcal{N}(0,1)$, the theorem follows.

\section{Concluding remarks}

It was proved in [4] that $m>>n^{3 / 2}$ is required for the concentration of $X_{n}$ in $\mathcal{G}(n, m)$, where $X_{n}$ denotes the number of Hamilton cycles or perfect matchings or spanning trees, as the variable will become asymptotically log-normally distributed when $m=\Theta\left(n^{3 / 2}\right)$. However, we do not think this condition is sufficient in the case of triangle-factors. It is surprising that the critical point of $m$ when $X_{n}$ changes from small deviation $\left(\mathbf{E} X_{n}^{2} \sim\left(\mathbf{E} X_{n}\right)^{2}\right)$ to large deviation $\left(\lim \sup _{n \rightarrow \infty} \mathbf{E} X_{n}^{2} /\left(\mathbf{E} X_{n}\right)^{2}>\right.$ 1 ) in $\mathcal{G}(n, m)$ seems to be different for Hamilton cycles and for triangle-factors. We guess $m=n^{5 / 3}$ might be the critical point for the latter case.

As explained in Section 4, the most interesting set $\mathscr{S}$ to be studied is perhaps the one containing graphs isomorphic to an unlabelled graph $H_{n}$ on $n$ vertices. Unfortunately, it is not easy to define the sequence $\left(H_{n}\right)_{n \geq 1}$ in general and for a general $H_{n}$, computing $r_{j}$ might be hard. It will be interesting to discover more classes of such graph sequences $\left(H_{n}\right)$ and see whether the corresponding random variables $X_{n}$ follow the log-normal paradigm. 


\section{References}

[1] A. Frieze and S. Suen, Counting the number of Hamilton cycles in random digraphs, Random Structures Algorithms 3 (1992), no. 3, 235-241.

[2] P. Gao, Distribution of the number of spanning regular subgraphs in random graphs. Random Structures Algorithms, (2012), in press, DOI: 10.1002/rsa.20418.

[3] S. Janson, Random regular graphs: asymptotic distributions and contiguity, Combin. Probab. Comput. 4 (1995), no. 4, 369-405.

[4] S. Janson, The numbers of spanning trees, Hamilton cycles and perfect matchings in a random graph, Combin. Probab. Comput. 3 (1994), 97-126.

[5] B.D. McKay, Asymptotics for symmetric 0-1 matrices with prescribed row sums, Ars Combinatoria 19A (1985), 15-25.

[6] A. Rucínski, Subgraphs of random graphs: a general approach, Random graphs '83 (Poznan, 1983), pp. 221-229, North-Holland Math. Stud. 118, North-Holland, Amsterdam, 1985.

[7] A. Ruciński, When are small subgraphs of a random graph normally distributed? Probab. Theory Related Fields, 78 (1988), no. 1, 1-10.

[8] R. W. Robinson and N.C. Wormald, Almost all cubic graphs are Hamiltonian, Random Structures Algorithms 3 (1992), no. 2, 117-125.

[9] R.W. Robinson and N. C. Wormald, Almost all regular graphs are Hamiltonian, Random Structures Algorithms 5 (1994), no. 2, 363-374.

[10] E. M. Wright, For how many edges is a graph almost certainly Hamiltonian? J. London Math. Soc. (2) 8 (1974), 44-48.

[11] E. M. Wright, For how many edges is a digraph almost certainly Hamiltonian? Proc. Amer. Math. Soc. 41 (1973), 384-388. 